\title{
Meta-analysis: Assocation between promoter hypermethylation of DAPK (Death-Associated Protein Kinase) and cervical cancer
}

\author{
Lao Duc Thuan ${ }^{1}$, Truong Kim Phuong ${ }^{1 *}$ \\ ${ }^{1}$ Ho Chi Minh City Open University, Vietnam \\ *Corresponding author: phuong.tk@ou.edu.vn
}

\begin{abstract}
ARTICLE INFO
DOI: $10.46223 / \mathrm{HCMCOUJS.}$ tech.en.9.1.350.2019

Received: April 18 ${ }^{\text {th }}, 2019$

Revised: May $7^{\text {th }}, 2019$

Accepted: May 21 ${ }^{\text {st }}, 2019$
\end{abstract}

Keywords:

cervical cancer, DAPK1, methylation, meta-analysis

\section{ABSTRACT}

Purpose: Death-associated protein kinase (DAPK or DAPK1) is an important tumor suppressor protein that is involved in the regulation of cell activities. The aberrant methylation of $D A P K$ promoter has been reported in patients with cervical cancer. However, the association between DAPK1 and cervical cancer was not always unification, in previous studies. Therefore, in the current study, a meta-analysis was performed for the association between $D A P K$ gene's promoter hypermethylated and cervical cancer.

Methods: A systematic literature analysis was conducted based on the previous studies published in PubMed, PubMed Central (NCBI), Google by using the following keywords: cervical cancer, cervical carcinoma, Methylation, by the end of January 2018. The association between DAPK promoter methylation and cervical cancer was evaluated by odds ratio (ORs) and 95\% confidence intervals (CI). To evaluate the potential sources of heterogeneity, the meta-regression analysis and subgroup analysis were conducted.

Results: A total of 21 case-control studies relevant to the association between DAPK1 gene's promoter methylation frequency and cervical cancer, including 1600 cancer cases and 1011 control cases (non-cancerous cases). The analysis results indicated that the characteristic of candidate gene's promoter methylation increased the cervical cancer risk through the calculation of $\mathrm{OR}$ value $(\mathrm{OR}=21.25 ; 95 \% \mathrm{CI}=8.73-52.97 ; p<$ 0.001; Random effect model). The association between DAPK1 gene's promoter hypermethylation was confirmed in all the subgroups analyses, including materials and assays methods, ethnicity. Furthermore, this association is higher in cervical squamous cell carcinoma than cervical adenocarcinoma and is a characteristic of late-stage disease.

Conclusion: The hypermethylated $D A P K 1$ gene's promoter 
was also one of the etiological factors, lead to cervical tumorigenesis.

\section{Introduction}

In addition to the infection with oncogenic human papillomavirus, which is the most significant risk factor in the etiology of cervical cancer, the epigenetic alterations could result in heritable gene silencing without changes to genetic sequences and are recognized as the important cause of human cervical cancer (Burd, 2003; Lu, Ma, \& Zhao, 2012; Yang, 2013). The epigenetic molecular mechanisms associated with human cervical cancer comprise a variety of alterations, including the silencing of Tumor Suppressor Genes (TSGs) by hypermethylation of $\mathrm{CpG}$ islands ( $\mathrm{Lu}$ et al., 2012; Yang, 2013). Many cellular pathways, including DNA repair, cell cycle, apoptosis, etc., are proven to be inactivated by the hypermethylation of CpG islands located in the promoter regions of many TSGs (Esteller, 2002; Le et al., 2017). Among the involved TSGs, the Death-associated protein kinase 1 (DAPK1) gene, located at 9q21.33, encodes the $160-\mathrm{kD}$ calmodulin dependent serine-threonine kinase involved in multiple cellular signaling pathways that trigger cell survival, apoptosis and autophagy (Cai, Xiao, Niu, \& Zhong, 2017; Raveh \& Kimchi, 2001). The decreased expression of $D A P K$ is associated with the methylation of gene promoter has been frequently reported in various types of human cancers, including cancer of the cervix (Cai et al., 2017; Leung et al., 2008; Niklinska et al., 2009; Wang et al., 2016). However, there are significant differences in the frequency of $D A P K$ promoter in patients with cervical cancer, due to different populations, sample sizes, methods for methylation analysis. Moreover, whether or not the methylation frequency of $D A P K$ gene's promoter is correlated with clinicopathological characteristics, including sex, stages, and invasion remains debated. Thus, we performed the systematic review and meta-analysis to summarize the current previous studies and evaluate the relationship between the methylation status of $D A P K$ promoter and cervical cancer as an epigenetic marker of cervical cancer risk.

\section{Materials and methods}

\subsection{Search strategy, inclusion and exclusion criteria of literature}

The systematic review of relevant literature was conducted by using many keywords for the literature search: methylation, DAPK1, Death-associated protein kinase 1, cervical cancer, etc. on four electronic databases: PUBMED, Web of Science, Embase database, updated to December 2018. The aim of the first selection was to identify studies that relevant to establish the association between $D A P K$ promoter methylation and cervical cancer. Accordingly, the studies were included if they satisfied the following inclusion criteria: (1) The investigation/ identification of $D A P K$ promoter methylation, which correlated with cervical cancer; (2) The correlation between $D A P K$ promoter methylation and clinicopathological features of FDB; (3) cohort design studies of $D A P K$ promoter methylation and cervical cancer; (4) the identification method was not excluded. Additionally, only studies written in English were included in the current study. Types of case reports, abstracts, reviews, and letters to the editor were eliminated.

\subsection{Data extraction, quality assessment and statistical analysis}

Two of the authors independently reviewed all the eligible studies. The data were 
abstracted the following information in a standard format, including the name of the first author, year of publication, the region of study subjects, age of patients, source/type of samples, detection method, clinical features of cervical cancer, the sample size of case and control groups. Finally, the third reviewer independently reviewed the relevant extract data from the eligible studies.

In the current study, all data were analyzed using Medcalc®2018. The frequency of $D A P K$ promoter methylation was observed in both case and control studies. The strength of the association between $D A P K$ promoter methylation and cervical cancer was evaluated by Odds Ratio (OR) and Risk Relative (RR) with 95\% confidence intervals (95\% CIs). The degree of association between $D A P K$ promoter methylation and clinical features was also evaluated by OR and RR with 95\% CIs. Heterogeneity across studies was measured using Cochran's Q-test (Higgins \& Thompson, 2002). The random-effects model was employed when the p-value was less than 0.05 in the Q-test, indicating the presence of substantial heterogeneity. The Cochran's Q-test only indicates the presence of heterogeneity, thus, we also reported $I^{2}$ statistic, which estimated the percentage of outcome variability that can be attributed to heterogeneity across studies (Higgins \& Thompson, 2002). Furthermore, subgroups analyses were conducted by histological stage (Squamous Cell Carcinoma - SCC, Adenocarcinoma - AC), source/type of samples (Biopsy, scrape, and urine), detection methods (Methylation Specific PCR - MSP, Realtime quantitative MSP - QMSP), ethnicity (Asian, European, American, and African).

\section{Results}

\subsection{Identified study and data characteristics}

A total of 158 studies were initially identified by a systematic literature search. After the duplicates and non-relevant studies were excluded by considering the title and abstract of studies, 61 articles with potentially relevant studies were further investigated by examining the full text. Finally, 23 studies, including 21 case-control studies and 2 cohort studies, were included in current meta-analysis after excluding studied that not met the inclusion criteria. The detailed processes of the systematic review and selections are given as a PRISMA flow chart, illustrated in Figure 1. 


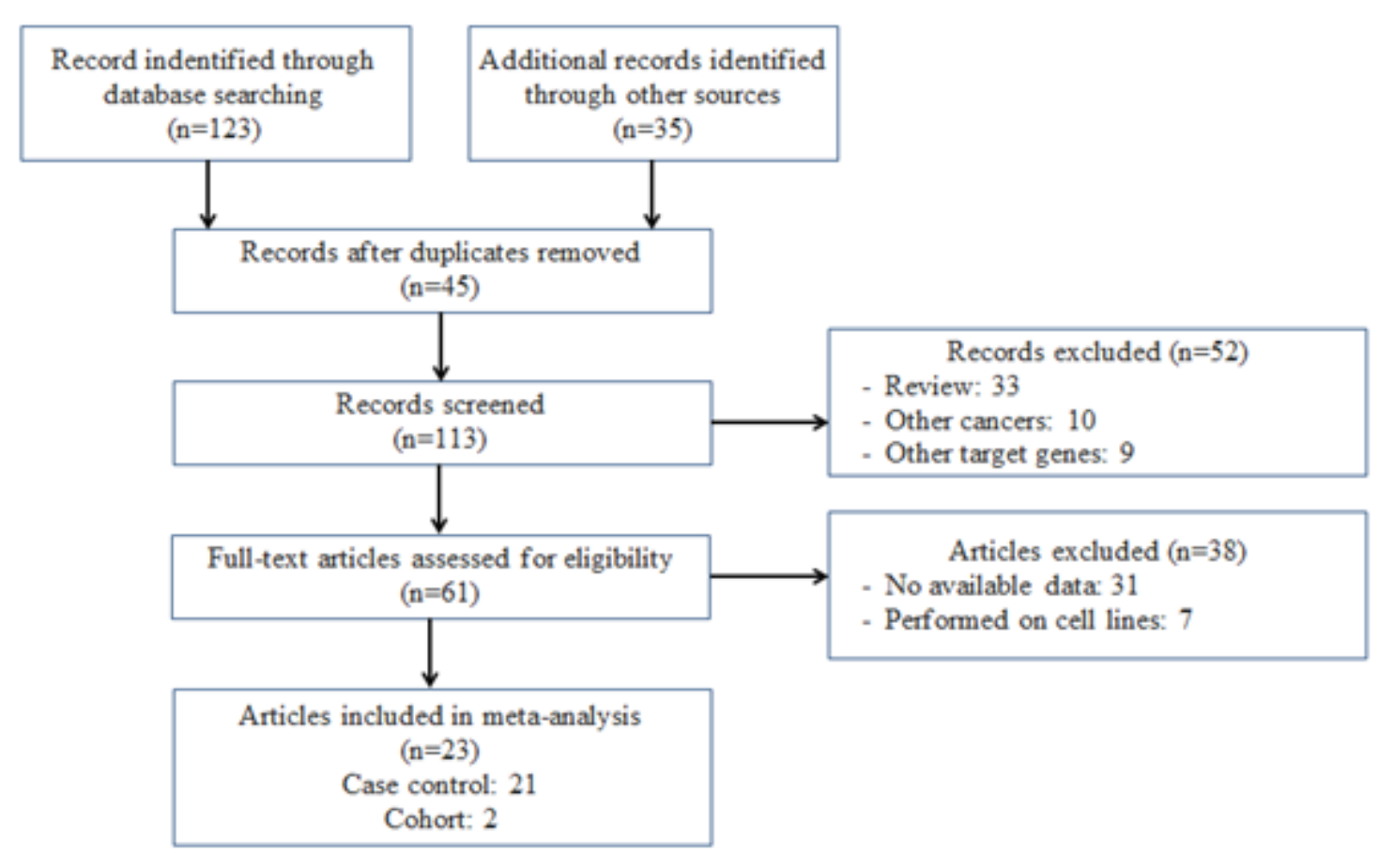

Figure 1. Flow chart of processes included in current meta-analysis

Out of the 23 studies included, 21 studies with 1600 cases and 1011 controls were combined to calculate the pooled OR between $D A P K$ promoter methylation and cervical cancer. The 21 case - control studies encompassed the publication years from 2001-2017, 11 from Asian countries (counting for 52.38\%), 5 from European countries (counting for 23.81\%), 3 from American countries (counting for 14.29\%), and 2 from Africa (counting for 9.52\%), were included in the systematic review and analysis. The methylation detection methods consisted of 15 studies of using MSP (counting for 71.43\%), 5 studies of using QMSP (counting for $23.81 \%$ ), and 1 study of using Realtime PCR (counting for $4.76 \%$ ), to explore DAPK promoter methylation in cervical cancer and corresponding to controls. The type of case samples consisted of 15 studies of using Biopsy - tumor tissues (counting for 71.43\%), 5 studies of using scrape sample (counting for $23.81 \%$ ), and 1 study of using urine (counting for $4.76 \%$ ). The type of controls consisted of 20 studies using normal/benign biopsy sample (counting for 95.24\%), and 1 study using healthy urine sample (counting for $4.76 \%$ ). The detailed characteristic of the included studies was summarized in Table 1.

\section{Table 1}

Detail characteristic of studies included in the systematic review and meta-analysis of $D A P K$ promoter methylation and cervical cancer

\begin{tabular}{|c|c|c|c|c|c|c|c|c|c|c|}
\hline \multirow{2}{*}{ First Author } & \multirow{2}{*}{ Year } & \multirow{2}{*}{ Country } & \multirow{2}{*}{ Ethnicity } & \multirow{2}{*}{ Method } & \multirow{2}{*}{$\begin{array}{c}\text { Types of } \\
\text { case }\end{array}$} & \multicolumn{2}{|c|}{ Cases } & \multirow{2}{*}{$\begin{array}{l}\text { Types of } \\
\text { control }\end{array}$} & \multicolumn{2}{|c|}{ Control } \\
\hline & & & & & & Total & Events & & Total & Events \\
\hline \multicolumn{11}{|c|}{ Case - control studies } \\
\hline Truong & 2017 & Vietnamese & Asian & MSP & Scrape & 61 & 27 & NCT & 48 & 1 \\
\hline Banzai & 2014 & Japan & Asian & MSP & Biopsy & 53 & 40 & NCT & 24 & 1 \\
\hline Niyaki & 2012 & China & Asian & MSP & Biopsy & 30 & 19 & NCT & 30 & 1 \\
\hline
\end{tabular}




\begin{tabular}{|c|c|c|c|c|c|c|c|c|c|c|}
\hline \multirow{2}{*}{ First Author } & \multirow{2}{*}{ Year } & \multirow{2}{*}{ Country } & \multirow{2}{*}{ Ethnicity } & \multirow{2}{*}{ Method } & \multirow{2}{*}{$\begin{array}{l}\text { Types of } \\
\text { case }\end{array}$} & \multicolumn{2}{|c|}{ Cases } & \multirow{2}{*}{$\begin{array}{l}\text { Types of } \\
\text { control }\end{array}$} & \multicolumn{2}{|c|}{ Control } \\
\hline & & & & & & Total & Events & & Total & Events \\
\hline Sun & 2012 & China & Asian & MSP & Biopsy & 331 & 181 & NCT & 336 & 157 \\
\hline Huang & 2011 & Taiwan & Asian & MSP & Scrape & 26 & 13 & NCT & 15 & 3 \\
\hline Missaoui & 2011 & Tunisia & African & MSP & Biopsy & 42 & 22 & NCT & 8 & 0 \\
\hline Kim & 2010 & Korea & Asian & MSP & Biopsy & 69 & 50 & NCT & 41 & 11 \\
\hline Yang & 2010 & Netherlands & European & QMSP & Biopsy & 60 & 31 & BCT & 20 & 5 \\
\hline Flatley & 2009 & UK & European & MSP & Scrape & 42 & 17 & $\mathrm{NCT}$ & 40 & 0 \\
\hline Iliopoulos & 2009 & Greece & European & RMethyLight & Biopsy & 61 & 41 & NCT & 15 & 0 \\
\hline Leung & 2008 & China & Asian & MSP & Biopsy & 107 & 60 & $\mathrm{NCT}$ & 27 & 0 \\
\hline Zhao & 2008 & China & Asian & MSP & Biopsy & 112 & 45 & NCT & 20 & 0 \\
\hline Feng & 2007 & Senegalease & African & QMSP & Urine & 63 & 31 & $\mathrm{U}$ & 16 & 1 \\
\hline Shivapurkar & 2007 & USA & American & QMSP & Biopsy & 45 & 24 & $\mathrm{NCT}$ & 12 & 0 \\
\hline Jeong & 2006 & Korea & Asian & MSP & Biopsy & 78 & 35 & NCT & 24 & 1 \\
\hline Wisman & 2006 & Netherland & European & QMSP & Scrape & 30 & 19 & NCT & 19 & 0 \\
\hline Feng & 2005 & USA & American & MSP & Biopsy & 176 & 69 & $\mathrm{NCT}$ & 140 & 3 \\
\hline Gustafson & 2004 & USA & American & MSP & Biopsy & 28 & 7 & $\mathrm{NCT}$ & 11 & 0 \\
\hline $\begin{array}{l}\text { Reesink- } \\
\text { Peters }\end{array}$ & 2004 & Netherlands & European & QMSP & Scrape & 48 & 35 & $\mathrm{NCT}$ & 41 & 2 \\
\hline Yang & 2004 & HK & Asian & MSP & Biopsy & 85 & 51 & $\mathrm{NCT}$ & 100 & 0 \\
\hline Dong & 2001 & Korea & Asian & MSP & Biopsy & 53 & 27 & $\mathrm{NCT}$ & 24 & 0 \\
\hline \multicolumn{11}{|l|}{ Cohort studies } \\
\hline Kalantari & 2014 & USA & American & MSP & Scrape & 408 & 317 & NA & NA & NA \\
\hline Henken & 2007 & Netherlands & European & MS-MLPA & Biopsy & 24 & 15 & NA & NA & NA \\
\hline
\end{tabular}

Note: Note: MSP: Methylation specific PCR; QMSP: Realtime quantitative MSP - QMSP; RMethyLight: Realtime MethyLight; NCT: normal cervical tissue; BCT: benign cervical tissue; U: Urine of healthy woman; NA: non-analysis

Source: The researcher's data analysis

Among the 2 included cohort studies consisted 432 cases, from American countries (1 of 2 studies, counting for 50\%) and European countries (1 of 2 studies, counting for 50\%), to estimate the frequency of $D A P K$ promoter methylation by using MSP ( 1 of 2 studies, counting for 50\%) and MS-MLPA (1 of 2 studies, counting for 50\%). The type of case samples consisted of 1 studies using Scrape samples (counting for 50\%), and 1 study using Biopsy - tumor tissues (counting for 50\%). The detailed characterization of the included studies was summarized in Table 1. 


\subsection{Meta-analysis}

\section{Association between $D A P K 1$ promoter methylation and cervical cancer}

In the current meta-analysis, the heterogeneity among included 21 case-control studies was significant for Cochran's Q-test ( $\mathrm{p}<0.001$ ), thus, the random effect model was applied to evaluate the strength of the association between DAPK promoter methylation and cervical cancer. The association was estimated by calculation of OR value with $95 \%$ confidence intervals $(95 \% \mathrm{CIs})$. As the results, we found that the frequency of DAPK promoter methylation was $52.75 \%$ (844 of 1600 cases), $18.40 \%$ (186 of 1011 controls) for cases and controls, respectively. Moreover, we found that $D A P K$ promoter methylation was significantly associated with an increased cervical risk with a pooled OR of $21.51(95 \% \mathrm{CI}=8.73-52.97)$ due to the significant heterogeneity $\left(\mathrm{I}^{2}=85.64 \%, \mathrm{p}<0.001\right)$, based on the random effect model (Figure 2).

\section{Subgroup analysis}

In the current study, subgroups analyses were performed by the source of sample types, methylation detection method and ethnicity. The association between DAPK1 promoter methylation and cervical cancer was observed in each subgroup (Table 2). The significant association between $D A P K$ promoter methylation and cervical cancer was identified including MSP and QMSP methods. The ORs were 21.41 (95\% CI = 7.03-65.19) in MSP subgroup and 20.45 (95\% CI $=5.61-74.51)$ in QMSP subgroup, based on the random effect model, while $I^{2}$ were high with $87.60 \%$ and $58.34 \%$, respectively. The subgroup analysis by the source of samples particularly focused on biopsy and scrape samples, reported that ORs were 20.30 (95\% $\mathrm{CI}=6.87-60.00)$ in case of using a biopsy, based on the random effect model, and 25.65 (95\% $\mathrm{CI}=11.25-58.44)$ in case of using Scrape sample, based on the fixed-effect model. According to subgroup analysis by ethnicity, a significant association between methylation status and cervical cancer was found among the Asian region and the non-Asian region, including American, European and African regions. In detail, the ORs were 20.93 (95\% CI = 5.77-75.97) in Asian region, 26.34 (95\% CI = 5.08-136.62) in European region, based on the random effect model, 25.48 (95\% CI = 9.16-70.92) in American region, and $15.88(95 \% \mathrm{CI}=2.92-86.41)$ in African region while $I^{2}$ were high with $85.50 \%$, moderate with $68.91 \%$, and low with both $0 \%$, respectively.

\section{Table 2}

Subgroup analysis in the meta-analysis of $D A P K$ promoter methylation and cervical cancer

\begin{tabular}{lccccccc}
\hline Variables & N & $\begin{array}{c}\text { Test of association } \\
\text { OR (95\% CI) }\end{array}$ & $\mathbf{Z}$ & P-value & Model & Ph & $\mathbf{I}^{\mathbf{2}} \mathbf{( \% )}$ \\
\hline Materials & & & & & & & \\
Biopsy & 15 & $20.30[6.87-60.00]$ & 5.44 & $<0.001$ & $\mathrm{R}$ & $<0.001$ & 87.11 \\
Scrape & 5 & $25.65[11.25-58.44]$ & 7.72 & $<0.001$ & $\mathrm{~F}$ & $<0.001$ & 47.96 \\
Urine & 1 & $14.53[18.07-116.74]$ & 2.52 & 0.01 & - & - & - \\
Methods & & & & & & & \\
MSP & 15 & $21.41[7.03-65.19]$ & 5.39 & $<0.001$ & $\mathrm{R}$ & $<0.0001$ & 87.60
\end{tabular}




\begin{tabular}{lccccccc}
\hline Variables & N & Test of association & & \multicolumn{5}{c}{ Test of heterogeneity } \\
OR (95\% CI) & Z & P-value & Model & Ph & $\mathbf{I}^{\mathbf{2}}(\%)$ \\
\hline QMSP & 6 & $20.45[5.61-74.51]$ & 4.57 & $<0.001$ & $\mathrm{R}$ & 0.03 & 58.34 \\
Ethnicity & & & & & & & \\
Asian & 11 & $20.934[5.77-75.97]$ & 4.63 & $<0.001$ & $\mathrm{R}$ & $<0.001$ & 85.50 \\
European & 5 & $26.34[5.08-136.62]$ & 3.90 & $<0.001$ & $\mathrm{R}$ & 0.001 & 68.91 \\
American & 3 & $25.48[9.16-70.92]$ & 6.20 & $<0.001$ & $\mathrm{~F}$ & 0.72 & 0.00 \\
Africa & 2 & $15.88[2.92-86.41]$ & 3.2 & 0.001 & $\mathrm{~F}$ & 0.89 & 0.00 \\
\hline
\end{tabular}

Source: The researcher's data analysis

\section{Discussion}

DNA hypermethylation of $\mathrm{CpG}$ islands located in the promoter of TSGs resulting in silencing the expression of TSGs that plays a crucial role in the carcinogenesis of the tumor. The $D A P K$ promoter methylation mainly induced the loss of $D A P K$ functions provides a unique mechanism that links suppression of apoptosis to metastasis of tumor, including cervical cancer (Cai et al., 2017; Inbal et al., 1997; Leung et al., 2008; Niklinska et al., 2009; Wang et al., 2016). The current results suggested that individuals with the DAPK methylation are associated with cervical cancer by calculating pooled OR of 21.51 (95\% CI $=8.73-52.97)$. 


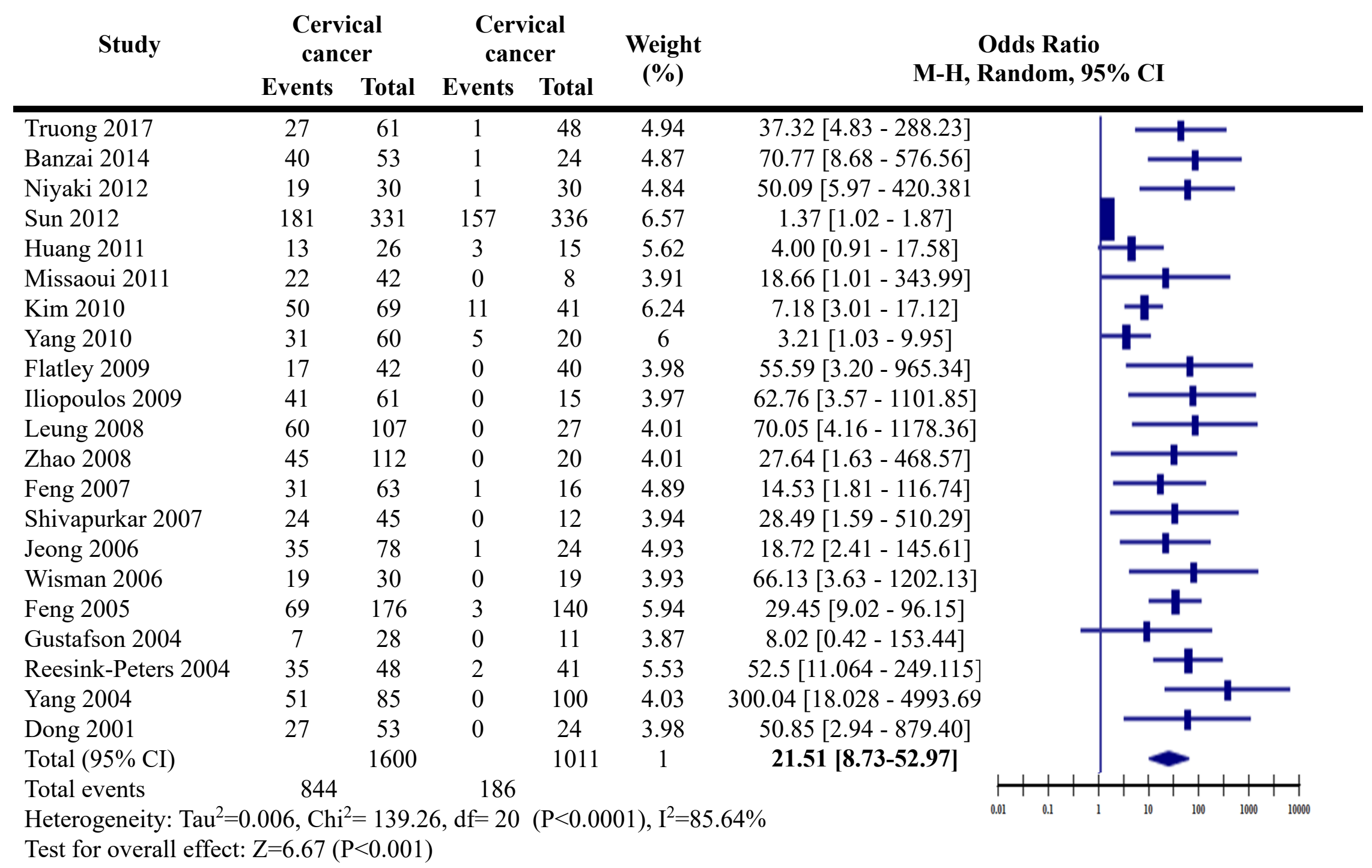

Figure 2. Forest plot of $D A P K 1$ promoter methylation and cervical cancer by calculating of OR value based on random effect model 
In our study reported that a meta-analysis of 21 published studies on 2611 samples, including 1600 cancerous samples and 1011 non-cancerous samples. As the results, we reported that the $D A P K$ would appear the promising methylated gene for cervical screening, which was similar to the previous study of Agodi, Barchitta, Quattrocchi, Maugeri, and Vinciguerra (2015) summarized the results of 20 published studies relevant to DAPK methylation, from 2001 to 2004, on 1929 samples. Because of the moderate heterogeneity between studies, the subgroup analyses by methylation detection method, sources of cancerous samples used to detect the $D A P K$ methylation were performed. In the methylation detection method, a significant association between $D A P K$ methylation and cervical cancer was observed between the MSP and QMSP in the random-effect method. The higher OR was observed in MSP method (MSP: OR $=21.41,95 \%$ CI = 7.03-65.19; QMSP: OR = 20.45, 95\% CI = 5.61-74.51). It could be explained that MSP method was considered to be "gold standard" method for methylation detection within reported results as methylated and/or unmethylated on the DNA sequence. The subgroup analysis by sources of samples revealed a significant association in subgroups, in detail, the OR was highly observed in both biopsy and scrape samples and the heterogeneity in the scrape subgroup was moderately low $\left(\mathrm{I}^{2}=47.96 \%\right)$. Finally, the DAPK methylation was the significant association revealed to ethnicity towards European country.

\section{Conclusion}

The methylation of $D A P K$ was reported as the epigenetic characteristic in many human cancers, including cervical cancer. The present meta-analysis provides evidence to conclude that the a significantly strong correlation between DAPK tumor suppressor gene hypermethylation in cervical cancer.

\section{References}

Agodi, A., Barchitta, M., Quattrocchi, A., Maugeri, A., \& Vinciguerra, M. (2015). DAPK1 promoter methylation and cervical cancer risk: A systematic review and a meta-analysis. PloS One, 10(8), 1-15.

Burd, E. M. (2003). Human papillomavirus and cervical cancer. Clinical Microbiology Reviews, 16(1), 1-17.

Cai, F., Xiao, X., Niu, X., \& Zhong, Y. (2017). Association between promoter methylation of DAPK gene and HNSCC: A meta-analysis. PloS One, 12(3), 1-13.

Esteller, M. (2002). CpG island hypermethylation and tumor suppressor genes: A booming present, a brighter future. Oncogene, 21(35), 5427-5440.

Higgins, J. P., \& Thompson, S. G. (2002). Quantifying heterogeneity in a meta-analysis. Statistics in Medicine, 21(11), 1539-1558.

Inbal, B., Cohen, O., Polak-Charcon, S., Kopolovic, J., Vadai, E., Eisenbach, L., \& Kimchi, A. (1997). DAP kinase links the control of apoptosis to metastasis. Nature, 390(6656), 180184.

Lao, T. D., \& Truong, P. K. (2017). DNA Hypermethylation in breast cancer. In Breast cancerfrom biology to medicine (pp. 147-161). Indianapolis, Indiana: IntechOpen. 
Le, D. T., Durham, J. N., Smith, K. N., Wang, H., Bartlett, B. R., Aulakh, L. K., ... Diaz, L. A. (2017). Mismatch repair deficiency predicts response of solid tumors to PD-1 blockade. Science, 357(6349), 409-413.

Leung, R. C. Y., Liu, S. S., Chan, K. Y. K., Tam, K. F., Chan, K. L., Wong, L. C., \& Ngan, H. Y. S. (2008). Promoter methylation of death-associated protein kinase and its role in irradiation response in cervical cancer. Oncology Reports, 19(5), 1339-1345.

Lu, Q., Ma, D., \& Zhao, S. (2012). DNA methylation changes in cervical cancers. In Cancer Epigenetics (pp. 155-176). Totowa, NJ: Humana Press.

Niklinska, W., Naumnik, W., Sulewska, A., Kozłowski, M., Pankiewicz, W., \& Milewski, R. (2009). Prognostic significance of DAPK and RASSF1A promoter hypermethylation in non-small cell lung cancer (NSCLC). Folia Histochemica et Cytobiologica, 47(2), 275280.

Raveh, T., \& Kimchi, A. (2001). DAP kinase-a proapoptotic gene that functions as a tumor suppressor. Experimental Cell Research, 264(1), 185-192.

Wang, W., Wang, J., Li, Z., Zhu, M., Zhang, Z., Wang, Y., \& Jing, H. (2016). Promoter hypermethylation of PTPL1, PTPN6, DAPK, p16 and 5-azacitidine inhibits growth in DLBCL. Oncology Reports, 35(1), 139-146.

Yang, H. J. (2013). Aberrant DNA methylation in cervical carcinogenesis. Chinese Journal of Cancer, 32(1), 42-48. 